\title{
Possible explanation for the tail of the cosmic ray spectrum
}

\author{
L.A. Anchordoqui ${ }^{\text {a }}$, M.T. Dova ${ }^{\text {b }, ~ T . P . ~ M c C a u l e y ~}{ }^{\text {a }}$, S. Reucroft ${ }^{\text {a }}$, J.D. Swain ${ }^{\text {a }}$

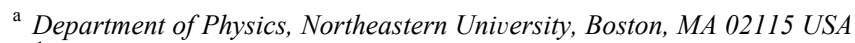 \\ ${ }^{\mathrm{b}}$ Departamento de Física, UNLP, C.C. 67 (1900) La Plata, Argentina
}

Received 10 April 2000; accepted 26 April 2000

Editor: W.-D. Schlatter

\begin{abstract}
A re-examination of the energy cosmic ray spectrum above $10^{20} \mathrm{eV}$ is presented. The overall data-base provides evidence, albeit still statistically limited, that non-nucleon primaries could be present at the end of the spectrum. In particular, the possible appearance of superheavy nuclei (seldom discussed in the literature) is analysed in detail. (C) 2000 Elsevier Science B.V. All rights reserved.
\end{abstract}

Keywords: Cosmic ray spectrum; High energy particle interaction

The origin and nature of cosmic radiation have been a constant source of mystery and discovery since 1949 [1]. Most notably, Greisen, Zatsepin and Kuz'min (GZK) pointed out that extremely high energy cosmic rays (usually assumed to be nucleons or nuclei) undergo reactions with the pervasive microwave background radiation (MBR) yielding a steep drop in their energy attenuation length [2]. Specifically, any proton energy above $50 \mathrm{EeV}$ is degraded by resonant scattering via $\gamma+p \rightarrow \Delta \rightarrow$ $p / n+\pi$, and heavy nuclei with energies above a few tens EeV get attenuated mainly by photodisintegration off the MBR and intergalactic infrared background photons (IR). Over the last few years, several giant air showers have been detected which confirm the arrival of particles with energies $\geq 100 \mathrm{EeV}$, this is, above the GZK cutoff (see [3] for a recent survey). Many models have been proposed as source candidates of such high energy events [4], however, it is not known for certain at the present time from where the rays originate.

In revealing their origin, the observed anisotropy of these cosmic rays is one of the most useful features. Very recently, the Fly's Eye [5] and Akeno Giant Air Shower Array (AGASA) [6] experiments reported a small but statistically significant anisotropy $\mathscr{O}(4 \%)$ in the cosmic ray flux towards the galactic plane at energies around $1 \mathrm{EeV}$. With increasing energy the picture looks rather different: although at $E>40 \mathrm{EeV}$ an enhancement of the flux from the Supergalactic plane was reported [7], the arrival directions above $100 \mathrm{EeV}$ are best described as isotropic, without imprint of correlation with the galactic plane or Supergalactic plane [8]. There are two extreme explanations for this puzzle: i) the bunch of nearby sources follows an isotropic distribution (which hardly could be the case) ii) One (A few) source(s) dominates at the highest energies 
whilst the background fields of the intergalactic medium strongly modify the particle propagation. For the latter explanation, it was suggested that a Galactic wind akin the solar wind could bend all the orbits of the highest energy cosmic rays towards the Virgo cluster (VC) [9]. Actually, if one assumes that these particles are protons, except for the two highest energy events (the one recorded at AGASA [10] and the super-GZK event reported by the Fly's Eye group [11]) all trajectories can be traced to within less than about 20 degrees from Virgo. ${ }^{1}$

At the highest energies, observed extensive air showers seem to be consistent with nucleon primaries but due to the poor statistics and large fluctuations from shower to shower an accurate determination of the particle species is not possible at the moment. Furthermore, extensive air shower simulations depend to some extent on the hadronic interaction event generator which complicates the interpretation of data even more [14]. Interestingly enough, however, the muon component of the highest AGASA event agrees with the expectation extrapolated from lower energies [10]. Indeed, a population of piled-up protons is expected at $50 \mathrm{EeV}$ [15], and the picture seems quite consistent. On the other hand, the Fly's Eye event occurs high in the atmosphere, and, although a primary proton cannot be excluded, a heavy nucleus more closely fits its shower development [16].

It is widely believed that the cosmic ray spectrum beyond the "crossover energy" (energy at which the local spectrum becomes comparable to or less than the cosmological component) could be associated with the presence of a particularly bright extragalactic, though relative nearby source, superimposed on a cosmological diffuse background. In Fig. 1 we show the evolved energy spectrum of nucleons assuming a cosmologically homogenous population of sources usually referred to as the universal hypothesis (UH)

\footnotetext{
${ }^{1}$ Notice that the highest energy Yakutsk event was excluded from this sample because of the great uncertainty on its energy determination. While first estimates suggested a primary energy around $120 \mathrm{EeV} \mathrm{[12],} \mathrm{a} \mathrm{re-estimation} \mathrm{of} \mathrm{the} \mathrm{number} \mathrm{of} \mathrm{charged}$ particles at $600 \mathrm{~m}$ from the shower core yields a possible primary energy of $300 \mathrm{EeV}$ [13].
}

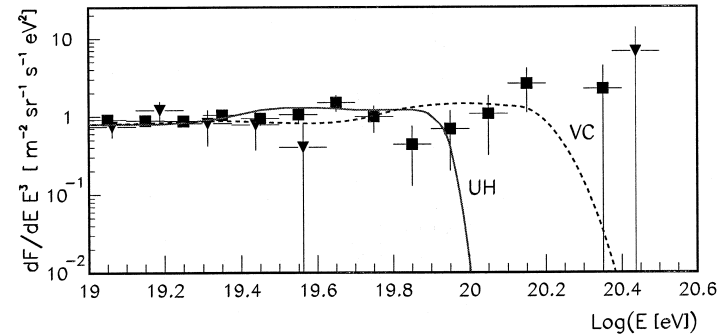

Fig. 1. The cosmic ray flux spectrum derived from AGASA (square) and Fly's Eye (triangle) experiments shown with the shape of the universal hypothesis (UH) spectrum (spectral index $\gamma=3.27$ [20]). We also show the expected flux of ultra high energy nucleons from the Virgo cluster (VC).

- [17], together with a compilation of recent air shower data [18]. In addition, we show the modified spectrum for the case of an extended source described by a Gaussian distribution of width $2 \mathrm{Mpc}$ at a distance of 18.3 Mpc (see [19] for details). Assuming that there is no other significant energy loss mechanism beyond interactions with the MBR for cosmic rays traversing parts of the cluster, this could be taken as a very crude model of Virgo. It is important to stress that for a galactic magnetic field $B_{\text {gal }}=7 \mu \mathrm{G}$ (as in [9]) which extends $R_{\text {halo }} \sim 1.5$ Mpc in the galactic halo, the mean flight time of the protons during their trip through the Milky Way is $\sim 5.05 \times 10^{6} \mathrm{yr}[21]$. This means that the bending does not add substantially to the travel time, and the continuous energy loss within the straight line approximation is expected to be reasonable for the problem at hand. From Fig. 1 we realize that the spectrum of the VC successfully reproduces the AGASA data above $100 \mathrm{EeV}$. However, it apparently cannot account for the super-GZK Fly's Eye event. The interpretation that we give for this result is that, without specific knowledge of the chemical composition, the best guess is that at the end of the spectrum two different types of characters are playing. $^{2}$

\footnotetext{
${ }^{2}$ We remark that AGASA data could be also reproduced if sources of ultra high energy protons trace the inhomogeneous distribution of luminous matter in the local present-epoch universe [22].
} 
At this stage, it is interesting to note that the measured density profile of the highest energy Yakutsk event (excluded in the analysis of [9]) shows a huge number of muons. Remarkably, its arrival direction coincides with the $300 \mathrm{EeV}$ Fly's Eye event, within angular resolution, possibly indicating a common origin. If this is the case, the almost completely muonic nature of this event, recently associated with a dust grain impact [23], could be, perhaps, the signature of a super-heavy nucleus.

It has been generally thought that ${ }^{56} \mathrm{Fe}$ is a significant end product of stellar evolution and higher mass nuclei are rare in the cosmic radiation. Strictly speaking, the atomic abundances of middle-weight $(60 \leq A<100)$ and heavy-weight $(A>100)$ elements are approximately 3 and 5 orders of magnitude lower, respectively, than that of the iron group [24]. The synthesis of the stable super-heavy nuclides is classically ascribed to three different stellar mechanisms referred to as the s-, r-, and p-processes. The s-process results from the production of neutrons and their capture by pre-existing seed nuclei on time scales longer than most $\beta$-decay lifetimes. There is observational evidence that such a kind of process is presently at work in a variety of chemically peculiar Red Giants [25] and in special objects like FG Sagittae [26] or SN1987A [27]. The abundance of well developed nuclides peaks at mass numbers $A=$ 138 and $A=208$. The neutron-rich (or r-nuclides) are synthesized when seed nuclei are subjected to a very intense neutron flux so that $\beta$-decays near the line of stability are far too slow to compete with the neutron capture. It has long been thought that appropriate r-process conditions could be found in the hot $\left(T \geq 10^{10} \mathrm{~K}\right)$ and dense $\left(\rho \sim 10^{10}-10^{11} \mathrm{~g} / \mathrm{cm}^{3}\right)$ neutron-rich (neutronized) material located behind the outgoing shock in a type II supernova event [28]. Its abundance distribution peaks at $A=130$ and $A=195$. The neutron-deficient (or p-nuclides) are 100-1000 times less abundant than the corresponding more neutron rich isobars, while their distribution roughly parallels the s- and r- nuclides abundance curve. It is quite clear that these nuclides cannot be made by neutron capture processes. It is generally believed that they are produced from existing seed nuclei of the s- or r-type by addition of protons (radiative proton captures), or by removal of neutrons (neutron photodisintegration). The explo- sion of the H-rich envelopes of type II supernovae has long been held responsible for the synthesis of these nuclides [24].

In light of the above, starbursts appear (hopefully) as the natural sources able to produce relativistic super-heavy nuclei. These astrophysical environments are supposed to comprise a considerable population of $\mathrm{O}$ and Red Giant stars [29], and we believe the supernovae rate is as high as $0.2-0.3 \mathrm{yr}^{-1}$ [30]. Of special interest here, the arrival directions of the Fly's Eye and Yakutsk super-GZK events $\left(b=9.6^{\circ}\right.$, $l=163^{\circ}$ and $\left.b=3^{\circ}, l=162^{\circ}\right)$ seem to point towards the nearby metally-rich galaxy $\operatorname{M} 82\left(b=41^{\circ}, l=\right.$ $\left.141^{\circ}\right)$ [31] which has been described as the archetypal starburst galaxy [32] and as a prototype of superwind galaxies [33]. The joint appearance of the galactic wind and the galactic magnetic field during particle propagation could certainly account for the required $37^{\circ}$ deflection. In addition, it was recently suggested that within this type of galaxies, iron nuclei can be accelerated to extremely high energies if a two step process is invoked [34]. In a first stage, ions are diffusively accelerated up to a few $\mathrm{PeV}$ at single supernova shock waves in the nuclear region of the galaxy [35]. Since the cosmic ray outflow is convection dominated, the typical residence time of the nuclei in the starburst results in $t \sim 1 \times 10^{11} \mathrm{~s}$. Thus, the total path traveled is substantially shorter than the mean free path (which scales as $A^{-2 / 3}$ ) of a super-heavy nucleus (for details see [34]). Those which are able to escape from the central region without suffering catastrophic interactions could be eventually re-accelerated to superhigh energies at the terminal shocks of galactic superwinds generated by the starburst. The mechanism efficiently improves as the charge number $Z$ of the particle is increased. For this second step in the acceleration process, the photon field energy density drops to values of the order of the cosmic background radiation (we are now far from the starburst region). The dominant mechanism for energy losses in the bath of the universal cosmic radiation is the photodisintegration process [36]. Notice that the energy loss rate due to photopair production could be estimated as $Z^{2} / A$ times higher than that of a proton with the same Lorentz factor, and thus could be safely neglected [37]. The disintegration rate $R$ (in the system of reference where the MBR is at $2.73 \mathrm{~K}$ ) of an ex- 
Table 1

Giant dipole resonance parameters

\begin{tabular}{lll}
\hline$\epsilon_{0}[\mathrm{MeV}]$ & $\sigma_{0}[\mathrm{mb}]$ & $\Gamma_{0}[\mathrm{MeV}]$ \\
\hline 13.15 & 255 & 2.9 \\
13.90 & 365 & 4.0 \\
\hline
\end{tabular}

tremely high energy nucleus with Lorentz factor $\Gamma$, propagating through an isotropic soft photon background reads [38], ${ }^{3}$

$$
R=\frac{1}{2 \Gamma^{2}} \int_{0}^{\infty} d \epsilon \frac{n(\epsilon)}{\epsilon^{2}} \int_{0}^{2 \Gamma \epsilon} d \epsilon^{\prime} \epsilon^{\prime} \sigma\left(\epsilon^{\prime}\right),
$$

where $\sigma$ stands for the total photon absortion cross section. The density of the soft photon background $n(\epsilon)$ can be modeled as the sum of: i) the MBR component which follows a Planckian distribution of temperature $\approx 2.73 \mathrm{~K}$, ii) the IR background photons as estimated in [39], iii) a black body spectrum with $T=5000 \mathrm{~K}$ and a dilution factor of $1.2 \times 10^{-15}$ to account for the optical (O) photons. The total photon absortion cross section is characterized by a broad maximum, designated as the giant resonance, located at an energy of 12-20 MeV depending on the nucleus under consideration. For the medium and heavy nuclei, $A \geq 50$, the cross section can be well represented by a single, or in the case of the deformed nuclei, by the superposition of two Lorentzian curves of the form

$\sigma\left(\epsilon^{\prime}\right)=\sigma_{0} \frac{\epsilon^{\prime 2} \Gamma_{0}^{2}}{\left(\epsilon_{0}^{2}-\epsilon^{\prime 2}\right)^{2}+\epsilon^{\prime 2} \Gamma_{0}^{2}}$.

In order to make some estimates, hereafter we refer our calculations to a gold nucleus (the resonance parameters are listed in Table 1 [40]). In Fig. 2 we show the ${ }^{197} \mathrm{Au}$ photodisintegration rate due to interactions with the starlight and relic photons. At the highest energies, the energy losses are dominated by collisions with the tail of $2.73 \mathrm{~K}$ Planckian spectrum. It is straightforward to show that a superheavy nucleus of a few hundred EeV emitted by M82 can traverse almost unscathed through the primeval radi-

\footnotetext{
${ }^{3}$ Primed quantities refer to the rest frame of the nucleus.
}

ation to produce an extensive air shower after interaction with the earth atmosphere.

Additional support for the superheavy nucleus hypothesis comes from the CASA-MIA experiment [41] (See in particular Figure 9). The collected cosmic ray data between $10^{14}-10^{16} \mathrm{eV}$ tends to favor a supernova shock wave acceleration scenario. The average mass increases with energy, becoming heavier above $10^{15} \mathrm{eV}$. At the maximum energy the results are consistent at $1 \sigma$ level with nuclei heavier than iron. However, "lore" has settled down some comparisons of the admittedly limited ultra high energy cosmic ray sample against hadronic-interaction-event generators which predicts the arrival of particle species heavier than iron $[41,42]$. We would like to stress that since simulations are used to interpret data, and then the data is used to modify the simulation, one has to be very careful and as we have shown, it is by no means clear that superheavy nuclei could not be present at the end of the spectrum.

The energy spectrum of nearby (around $3 \mathrm{Mpc}$ ) nuclear sources was discussed elsewhere [43]. The analysis showed that particles tend to pile up between 240-270 EeV. This bump-like feature is followed by a simultaneous drop in the cosmic ray flux of the preceding bins of energy, changing the relative

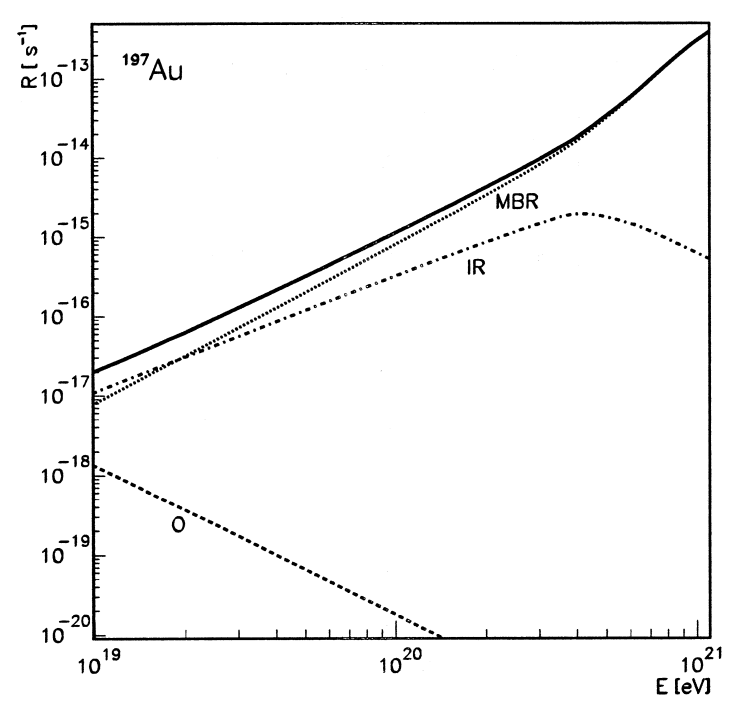

Fig. 2. Fractional energy loss for ${ }^{197} \mathrm{Au}$ photodisintegration on MBR, IR, O, as well as the total (solid line). 
detection probabilities. As a consequence particles in the pile-up are $50 \%$ more probable than those at lower energies.

In summary, the recently reported AGASA data can be successfully reproduced by a power law spectrum of nucleons hailing from the VC superimposed on a cosmological diffuse background. The Fly's Eye observations may also fit this scenario, albeit with large errors. One might also consider less likely astrophysical sources. In particular, our analysis seems to indicate that the next-door galaxy M82 could be responsible for some events at the end of the CR spectrum. This has also been suggested elsewhere $[31,34,43]$. At least some of these superGZK events could be due to heavy, and even superheavy nuclei. Clearly, more data is needed before this hypothesis can be verified. In this regard, the coming avalanche of high quality cosmic ray observations at the Southern Auger Observatory [44] will provide new insights to the ideas discussed in this letter.

Note added: After we finished this work, it was argued that the Galactic wind model assumed in Ref. [9] is alone responsible for the focusing of positive particles towards the North galactic pole. Therefore the apparent clustering of the back-traced CR cannot be interpreted as evidence for a point source, this point source identified as M87 [45]. It should be pointed out that the main input parameters for the determination of the CR-spectrum in Fig. 1 are the spectral index of the source, and the propagation distance of the nucleons in the extragalacic medium. The Galactic wind model is just used to collect all the traces in only one single direction in the sky. Therefore the discussion presented in this letter strongly supports older suspicions regarding M87, like the model proposed in Ref. [9].

\section{Acknowledgements}

In closing, we wish to thank Gustavo Medina Tanco for a fruitful discussion. The research of LAA was supported by CONICET. MTD was supported by CONICET and Fundación Antorchas. TPM-SRJDS were supported by the National Science Foundation.

\section{References}

[1] J.R. Oppenheimer, Rev. Mod. Phys. 21 (1949) 181.

[2] K. Greisen, Phys. Rev. Lett. 16 (1966) 748; G.T. Zatsepin, V.A. Kuz'min, Pis'ma Zh. Éksp. Teor. Fiz. 4 (1966) 114 [JETP Lett. 4 (1966) 78].

[3] S. Yoshida, H. Dai, J. Phys. G 24 (1998) 905.

[4] P. Bhattacharjee, G. Sigl, astro-ph/9811011; and references therein.

[5] D.J. Bird et al., Astrophys. J 511 (1999) 739.

[6] N. Hayashida et al., Astropart. Phys. 10 (1999) 303.

[7] T. Stanev, P.L. Biermann, J. Lloyd Evans, J.P. Rachen, A.A. Watson, Phys. Rev. Lett. 75 (1995) 3056.

[8] A.M. Hillas, Nature 395 (1998) 15.

[9] E.J. Ahn, G. Medina Tanco, P.L. Biermann, T. Stanev, The origin of the highest energy cosmic rays: Do all roads lead back to Virgo?, astro-ph/9911123.

[10] N. Hayashida et al., Phys. Rev. Lett. 73 (1994) 3491.

[11] D.J. Bird et al., Phys. Rev. Lett. 71 (1993) 3401; Astrophys. J 441 (1995) 144.

[12] N.N. Efimov et al., in: Astrophysical Aspects of the Most Energetic Cosmic Rays, M. Nagano, F. Takahara (Eds.), World Scientific 1191), p. 20 - p. 434.

[13] E.E. Antonov et al., Pis'ma Zh. Éksp. Teor. Fiz. 69 (1999) 614 [JETP Lett. 69 (1999) 650].

[14] L.A. Anchordoqui, M.T. Dova, S.J. Sciutto, in: Proc. $26^{\text {th }}$ International Cosmic Ray Conference, D. Kieda, M. Salamon, B. Dingus (Eds.), Salt Lake City, Utah, 1999), vol. I, p. 147; L.A. Anchordoqui, M.T. Dova, L.N. Epele, S.J. Sciutto, Phys. Rev. D 59 (1999) 094003.

[15] C.T. Hill, D. Schramm, Phys. Rev. D 31 (1985) 564; V.S. Berezinsky, S.I. Grigor'eva, Astron. Astrophys. 199 (1988) 1.

[16] F. Halzen, R.A. Vázquez, T. Stanev, H.P. Vankov, Astropart. Phys. 3 (1995) 151

[17] To work through the cosmological spectrum we follow the analytical approach introduced in F.W. Stecker, Nature 342 (1989) 401. See specifically Eq. (8). Numerical analyses also support our results, S. Yoshida, M. Teshima, Prog. Theor. Phys. 89 (1993) 833.

[18] M. Takeda et al., Phys. Rev. Lett. 81 (1998) 1163; astroph/9902239; D.J. Bird et al., Phys. Rev. Lett. 71 (1994) 3401.

[19] L.A. Anchordoqui, M.T. Dova, L.N. Epele, J.D. Swain, Phys. Rev. D 55 (1997) 7356.

[20] The selected spectral index for the UH and VC spectra was taken from the fit to Fly's Eye stereo data in the region below the photo pion-production threshold, where no modifications on the spectrum are expected. M.T. Dova, L. Epele, C. Hojvat, in: Proc. $25^{\text {th }}$ International Cosmic Ray Conference, M.S. Potgieter, B.C. Raubenheimer, D.J. van der Walt (Eds.), Durban, South Africa, 1997) v. 7, p. 381.

[21] G. Medina Tanco, private communication.

[22] G. Medina Tanco, Astrophys. J 510 (1999) L91.

[23] L.A. Anchordoqui, Phys. Rev. D 61 (2000) 087302.

[24] E.M. Burbidge, G.R. Burbidge, W.A. Fowler, F. Hoyle, Rev. Mod. Phys. 29 (1957) 547. 
[25] V.V. Smith, in: Cosmic Abundances of matter, AIP Conference Proc. 183, C.J. Waddington (Ed.), (New York, AIP 1989), p. 200.

[26] G.E. Langer, R.P. Kraft, K.S. Anderson, Astrophys. J 189 (1974) 509.

[27] P.A. Mazzali, L.B. Lucy, K. Butler, Astron. Astrophys. 258 (1992) 399.

[28] M.D. Delano, A.G.W. Cameron, Astrophys. Space. Sci. 10 (1971) 203; W. Hillebrandt, K. Takahashi, T. Kodama, Astron. Astrophys. 52 (1976) 63.

[29] S. Sakai, B. Madore, Detection of the Red Giant Branch Stars in M82 Using the Hubble Space Telescope, astro$\mathrm{ph} / 9906484$.

[30] See for instance, T.W.B. Muxlow et al., Mont. Not. Roc. Astron. Soc. 266 (1994) 455; J.S. Ulvestad, R.J. Antonucci, Astrophys. J 448 (1997) 621; D.A. Forbes et al., Astrophys. J 406 (1993) L11; R. de Grijs et al., Supernova Remnants in the Fossil Starburt in M82, astro-ph/9909044.

[31] J.W. Elbert, P. Sommers, Astrophys. J 441 (1995) 151.

[32] A.J. Fitt, P. Alexander, Mont. Not. Roc. Astron. Soc. 261 (1993) 445.

[33] T.H. Heckman, L. Armus, G.K. Miley, Astrophys. J 74 (1990) 833
[34] L.A. Anchordoqui, G.E. Romero, J.A. Combi, Phys. Rev. D 60 (1999) 10300.

[35] P.O. Lagage, C.J. Cesarsky, Astron. Astrophys. 125 (1983) 249.

[36] J.L. Puget, F.W. Stecker, J.H. Bredekamp, Astrophys. J 205 (1976) 638; L.N. Epele, E. Roulet, J. High Energy Phys. 10 (1998) 009; F.W. Stecker, M.H. Salamon, Astrophys. J 512 (1999) 521

[37] G. Blumenthal, Phys. Rev. D 1 (1970) 1596; M.J. Chodorowski, A.A. Zdziarski, M. Sikora, Astrophys. J 400 (1992) 181.

[38] F.W. Stecker, Phys. Rev. D 180 (1969) 1264.

[39] M.A. Malkan, F.W. Stecker, Astrophys. J 496 (1998) 13

[40] E.G. Fuller, M.S. Weiss, Phys. Rev. 112 (1958) 560.

[41] M.A.K. Glasmacher et al., Astropart. Phys. 12 (1999) 1.

[42] M. Nagano, D. Heck, K. Shinozaki, N. Inoue, J. Knapp, Comparison of AGASA data with CORSIKA simulation, astro-ph/9912222. See also Ref. [13]

[43] L.A. Anchordoqui, M.T. Dova, L.N. Epele, J.D. Swain, Phys. Rev. D 57 (1998) 7103.

[44] http://www.auger.org.

[45] P. Billoir, A. Letessier-Selvon, Note on the Origin of the Highest Energy Cosmic Rays, astro-ph/0001427. 